\title{
Measures of journal quality should separate reviews from original research
}

\author{
Sarah R. Supp and Ethan P. White \\ S.R. Supp (sarah.supp@usu.edu) Department of Biology and The Ecology Center, Utah State University 5305 Old \\ Main Hill, Logan, UT 84322-5305 \\ E.P. White (ethan.white@usu.edu) Department of Biology and The Ecology Center, Utah State University 5305 Old \\ Main Hill, Logan, UT 84322-5305
}

\begin{abstract}
Metrics of journal quality (e.g., impact factors) are often used to make important judgments regarding journal quality and importance. It is well known that reviews are more highly cited than original research articles. Therefore, it is not surprising that review journals within a field tend to have the highest scores on measures of journal impact/quality. However, many journals publish both reviews and original research, which may lead to a misleading ranking system because published metrics are a mixture of two potentially independent measures with different means. In addition, journals under pressure to increase their impact factors have suggested that changing publication practices to include more reviews is a legitimate manipulation. However, the proportion of reviews published is not directly related to journal quality. Using 20 top ecology journals, we measure the influence of reviews on impact factor and clearly show that the proportion of reviews published by a journal can explain greater than $75 \%$ of the observed variability in measures of journal quality. We suggest that these measures will be more useful if they are reported separately for articles and reviews. In contrast to other articles published on the problems with impact factors, we suggest a clear, simple solution that could be readily instituted with little change to the existing system.
\end{abstract}

Keywords: impact factor, citation metrics, review, article, ecology
Impact factors and other metrics are often used to make judgments regarding journal quality and article importance. Much has been written about the challenges of quantifying scientific value and the problems with commonly used journal metrics. Although originally intended as a measure of the scientific significance of a journal (i.e., how often articles within a journal are cited to communicate original research, Garfield 1972), impact factors have come under fire recently for being overemphasized (Nature editors 2005), leading to the pursuit of "trendy" science (Statzner et al 1995), and being unfairly used to compare progress among related fields (Kokko and Sutherland 1999, Metcalfe 1995, Moed 2005, Taborsky 2007, Postma 2007).

Most measures of journal quality are calculated based on papers loosely classified as "articles", "reviews", and "proceedings" (Amin and Mabe 2000). Since it is well-known that reviews are more highly cited than original research articles (Moed 2005, Nature editors 2005, PLoS Medicine editors 2006), it is not surprising that most top ranked journals within a field do not report new research findings (Hecht et al 1998, Altmann and Gorman 1998, Ketcham and Crawford 2007). In addition to being more "citable", reviews also have a higher immediacy factor, meaning that they are frequently cited more often and more quickly (i.e. within the short windows typically used to calculate measures of journal quality) than research articles (Amin and Mabe 2000). When a journal only publishes reviews this does not cause confusion because it is readily recognized that this policy largely determines the high impact factor. However, this distinction becomes less clear when a 
journal publishes both reviews and original research. Specifically, confusion may occur because measures of journal quality/impact that include both kinds of articles will be influenced by three things: 1) the impact of the primary research; 2) the impact of the reviews; and 3) the proportion of reviews published. The first two of these are related to the quality of the papers being published, but the third is simply an editorial decision regarding what fraction of each type of paper to publish. As such, the third factor is by definition not directly related to the quality of the published articles.

Because reviews tend to be highly cited, journals publishing a higher proportion of reviews may appear to be higher quality based solely on their high impact factor. If journals make publishing decisions based partly on increasing impact factors, there may be pressure to publish a higher proportion of reviews (Garfield 1996, Ketcham and Crawford 2007). In fact, it has been suggested that publishing more reviews is a legitimate manipulation of impact factors that many journals engage in (Tempest 2008). However, as described above, the proportion of reviews is not related to the quality of published articles; it is simply an editorial choice. Here we demonstrate the potential influence of reviews on citations metrics using data from the major ecology journals and suggest that measures of journal quality will be more useful if they are reported separately for articles and reviews.

Citation data was obtained from the Web of Science (WoS; http://isiknowledge.com) for papers published from 2001-2006 in the top 20 ecology journals (based on 2006 impact factors). WoS categorizes a paper as a review if it has more than 100 references, appears in a review journal or the review section of a research journal, or states in the abstract that it is a review (Ketcham and Crawford 2007). Our analysis included citation data from 17,134 articles including 1,401 reviews. Impact factors for 20032007 were calculated using the standard formula: the average number of times an article published in year $t-1$ and year $t-2$ were cited in year $t$. We chose the impact factor as the measure of journal quality because it is the most commonly used metric, but our results should hold using alternative metrics such as the Article Influence Score (http://www.eigenfactor. org). Published Journal Citation Reports Impact Factors are based on the journal in which a paper is published, whereas WoS citation numbers are based on individual articles (Ann Kusmerick, personal communication). As a result, our impact factors deviate slightly from those published by Journal Citation Reports, but they are strongly correlated $(r=0.93)$ and this minor difference should not influence our results. All interpretations of these results are subject to the

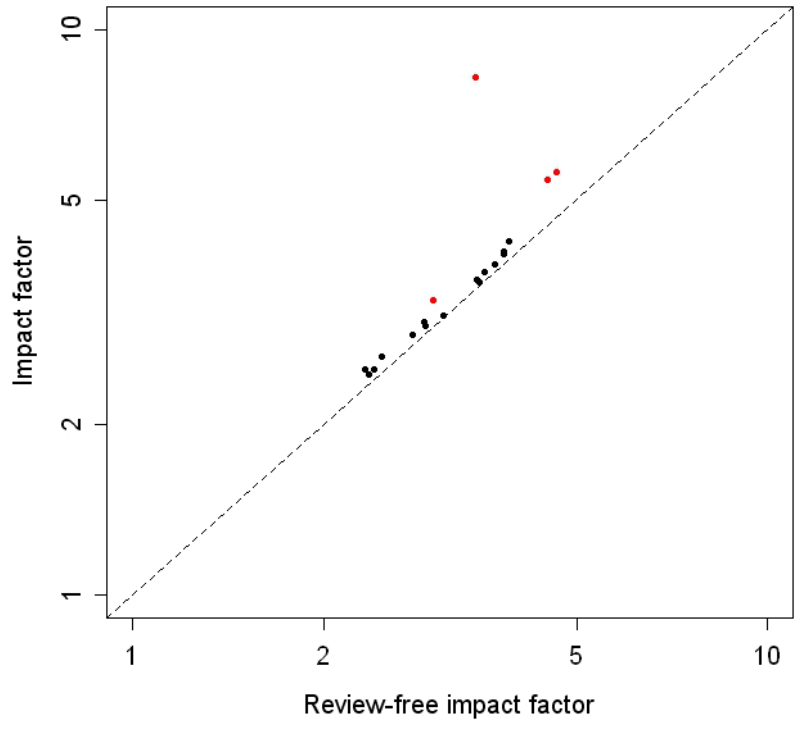

Figure 1. Mean review-free impact factor is plotted against mean impact factor. A Wilcoxon-signed rank test on the differences between mean impact factor and mean review-free impact factor across years 2003-2007, demonstrated a significant difference $(\mathrm{p}<0.0001)$. Red points represent journals publishing $>10 \%$ review articles. One journal, Annual Review of Ecology, Evolution, and Systematics is not represented in this plot and was not included in the statistical analysis because it publishes only reviews (and therefore does not have a review-free impact factor). Axes are logarithmically scaled.

usual caveats related to citation based metrics of journal quality. There are many reasons an article may be cited: it may be high quality, or it may be authored by prestigious researchers or someone with whom the citer has a personal relationship, contain a flawed design, or simply be a matter of convenience, as is often the case with reviews (Cozzens 1989). Therefore, although high quality articles are often highly cited, the converse is not necessarily true. Regardless, given that these metrics are used as measure of quality it is important that the influence of mixing reviews and original research be considered.

Our results demonstrate a significant difference between impact factors calculated using all published articles and calculated when excluding reviews. A Wilcoxon-signed rank test of the difference in mean impact factor and mean review-free impact factor across the 5 years (2003-2007) showed a significant difference $(p<0.0001$, Figure 1$)$. In addition, there is a significant positive relationship between the proportion of reviews published and journal impact factor and rank, with the proportion of reviews explaining $77 \%$ of the variance in impact factor and 

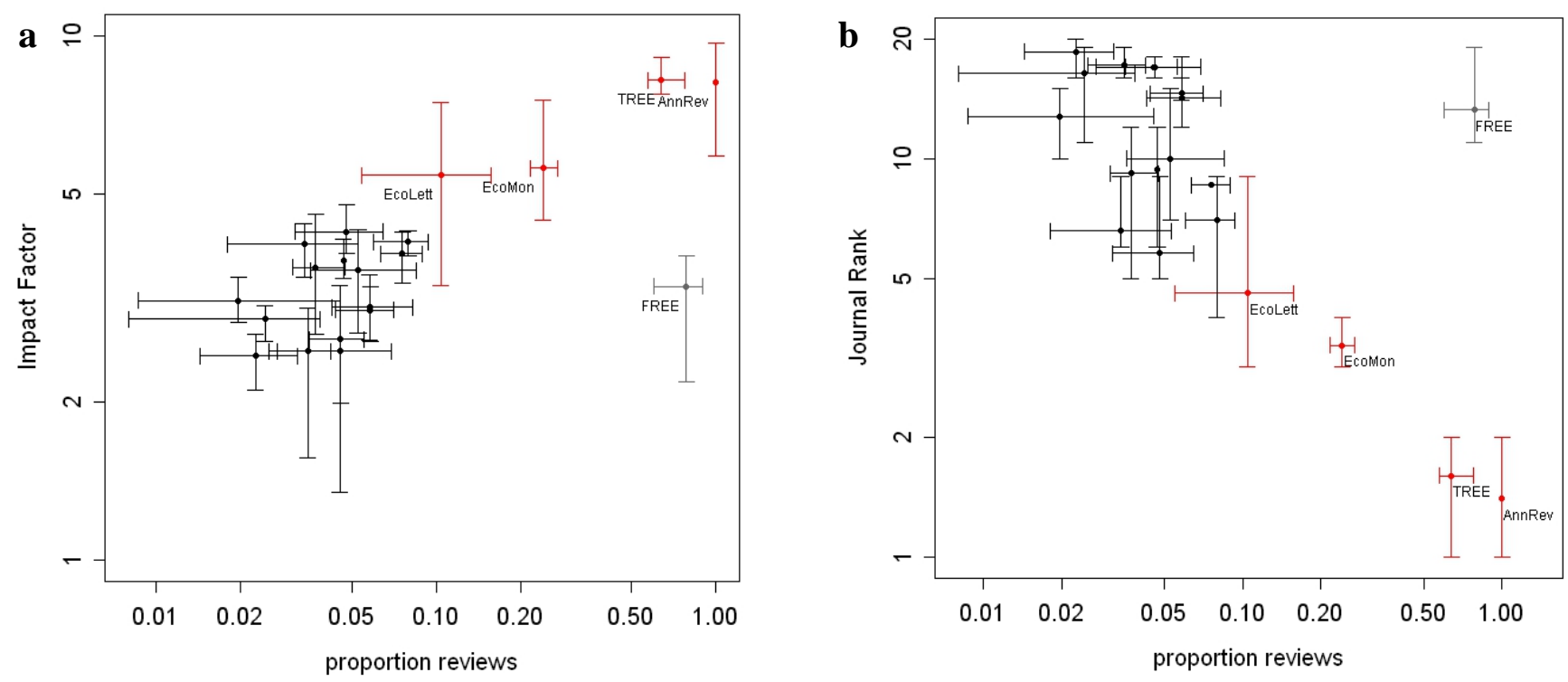

Figure 2. (a) Higher proportions of reviews (averaged across years) are associated with higher overall impact factor $\left(\mathrm{r}^{2}=0.77\right)$. The four journals labeled in red (Annual Review of Ecology, Evolution, and Systematics (AnnRev), Trends in Ecology and Evolution (TREE), Ecological Monographs (EcoMon), and Ecology Letters (EcoLett)) all publish more than 10\% review articles. Frontiers in Ecology and the Environment (FREE), in gray, also publishes more than $10 \%$ review articles, but was a significant outlier based on studentized residuals $(\mathrm{p}<0.0001)$, a standard approach to flag outliers. Points represent means, bars represent ranges (minimum and maximum). Axes are logarithmically scaled. (b) Higher proportions of reviews are also associated with higher mean journal rank $\left(\mathrm{r}^{2}=0.79\right.$, journal rank on scale of 1-20, highest to lowest, averaged across years). Points and bars are used in the same manner as Figure 2a.

$79 \%$ of the variance in journal rank with respect to impact factor (Figure 2).

We also calculated the average number of rank shifts for journals, comparing between impact factor and review-free impact factor. Journal rank was calculated for each year (2003-2007) among the 20 journals on a scale of 1-20, with 1 being highest and 20 being lowest, using journal impact factor. Journals publishing less than $10 \%$ review articles only shifted an average of 1.13 rank places when the impact factor calculation excluded reviews. However, journals publishing more than $10 \%$ reviews shifted an average of 4.89 rank places when reviews were omitted from impact factor calculation. It is clear that mixing reviews and original research can significantly influence a journal's impact factor and that differences in the proportion of reviews are partially responsible for differences in impact factor and other measures of journal quality among ecology journals.

One journal in particular, Ecology Letters, illustrates the potential importance of this issue. This journal has been steadily increasing the proportion of reviews it publishes and it appears that this increase and the resulting high proportion of reviews play a role in its steadily increasing impact factor (Figure 3).
For example, in 2003-2005, when Ecology Letters published a low percentage of reviews, reviews only elevated the impact factor by approximately 0.5 each year above that for original research. However, in 2006 and 2007 when the journal began to publish a higher percentage of reviews, reviews elevated the impact factor by approximately 1.5 compared to the value for original research.

Implementing strategies to increase measures of journal quality, which are used widely in journal advertisements (Seglen 1997), is underway in many publishing houses (Hecht et al 1998, PLoS Medicine editors 2006), and some journals are making explicit efforts to include more reviews: "We especially want to increase the frequency with which we provide synthetic reviews of topics of broad ecological interest" (Fox et al 2008: 1). Unfortunately, altering the number of reviews published in original research journals only obfuscates attempts to quantify the importance of both original research and review articles.

We suggest a simple change that would allow ecologists and other scientists to make more informed comparisons among journals within their field. By separating article and review impact factors for 


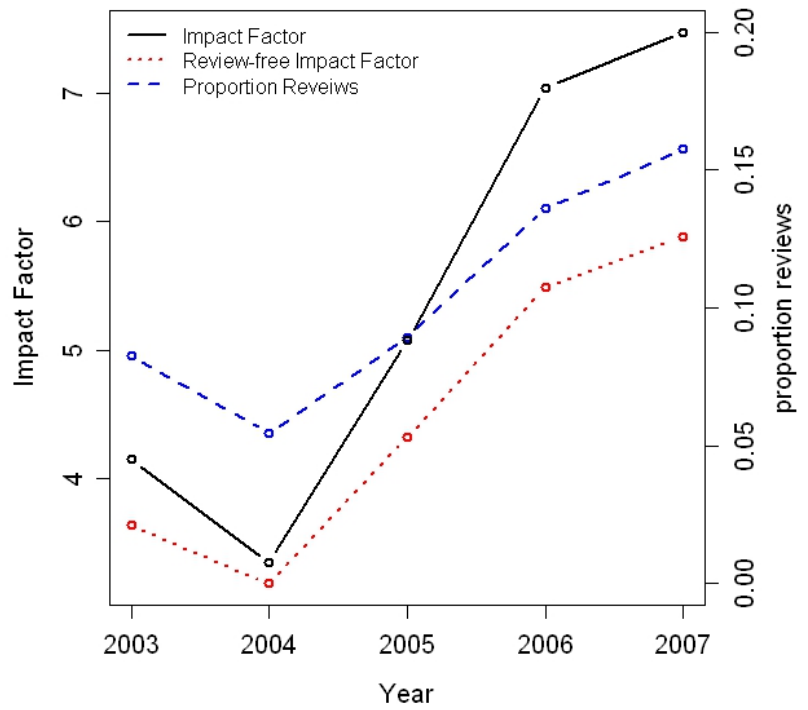

Figure 3. Ecology Letters has been increasing the proportion of reviews it includes from less than $10 \%$ to over $15 \%$ over the course of 5 years. Its overall impact factor shows a corresponding increase that is more rapid than would be expected from the increase in the original research impact factor alone (as demonstrated by the fact that the slope of the overall impact factor increase is steeper than that of the impact factor calculated by excluding reviews).

publications, it will be possible to more clearly understand the manner in which journals contribute to the scientific community through these distinct modes of communication. This requires no complicated calculations or new assumptions; it simply makes an important distinction between articles and reviews based on different citation practices for each publication type. Calculating measures of journal quality separately for research articles and reviews also eliminates the incentive for journals to alter publication practices. The fact that more than $75 \%$ of the observed variability in impact factor and impact factor rank can be explained by the proportion of reviews published by a journal clearly emphasizes that-as calculated-these measures may tell us more about editorial decision making, than about the quality of scholarship published by a journal.

\section{Referees}

Erik Postma - postma.erik@gmail.com

University of Zurich, Switzerland

John Parker - parker@nceas.ucsb.edu

NCEAS, United States

\section{References}

Altmann, K.G. and G. E. Gorman. 1998. The usefulness of impact factors in serial selection: A rank and mean analysis using ecology journals. Library Acquisitions: Practice and Theory 22:147159. CrossRef

Amin, M. and M. Mabe. 2000. Impact factor: use and abuse. Perspectives in Publishing 1:1-6.

Cozzens, S. E. 1989. What do citations count? The rhetoric-first model. Scientometrics 15:437-457.

Fox, C., Thompson K., Cresswell J., Messina F., and L. Baker. 2008. Editorial. Functional Ecology 22:12. CrossRef

Garfield, E. 1972. Citation analysis as a tool in Journal evaluation. Science 78:471-479. CrossRef

Garfield, E. 1996. How can impact factors be improved? British Medical Journal 313:411-413.

Hecht, F., Hecht, B.K., and A. A. Sandberg. 1998. The journal "impact factor": A misnamed, misleading, misused measure. Cancer Genetics and Cytogenetics 104:77-81. CrossRef

Ketcham, C. M. and J. M. Crawford. 2007. The impact of review articles. Laboratory Investigation 87:1174-1185. CrossRef

Kokko, H. and W. J. Sutherland. 1999. What do impact factors tell us? Trends in Ecology and Evolution 14:382-384. CrossRef

Metcalfe, N.B. 1995. Journal impact factors. Nature 376:720. CrossRef

Moed, H.F. 2005. Citation analysis of scientific journals and journal impact measures. Current Science 89:1990-1996.

Nature editors. 2005. Not so deep impact. Nature 435:1003-1004.

PLoS Medicine Editors. 2006. The Impact Factor Game. PLoS Medicine 3:e291. CrossRef

Postma, E. 2007. Inflated impact factors? The true impact of evolutionary papers in non-evolutionary journals. PLoS One 10:e999. CrossRef

Seglen, P. O. 1997. Why the impact factor should not be used for evaluating research. British Medical Journal 314:498-502.

Statzner, B., Resh, V. H. and N. G. Kobzina. 1995. Low impact factors of ecology journals: don't worry. Trends in Ecology and Evolution 10:220. CrossRef

Taborsky, M. 2007. Impact factor statistics and publication practice: What can we learn? Ethology 113:1-8. CrossRef

Tempest, D. 2008. Interview by Thomson Reuters. "Using and Misusing the Impact Factor". http://science.thomsonreuters.com/news/200803/8444405/ 